\title{
Evidence for Binary Orbital Motion of a Quasar Broad-Line Region
}

\author{
C. Martin Gaskell \\ Department of Physics \& Astronomy, University of Nebraska, Lincoln, NE 68588-0111
}

\begin{abstract}
Analysis of spectra of the quasar 3C 390.3 covering a period of over $20 \mathrm{yr}$ shows that the blueshifted peak of $\mathrm{H} \beta$ has been changing its radial velocity at an almost constant rate during this time. The radial velocity has increased by over $1500 \mathrm{~km} \mathrm{~s}^{-1}$. The lower limit to the period of radial velocity changes is 210 yr. Although very long periods cannot be excluded by the radial velocity curve alone, other considerations suggest that the period is $\sim 300 \mathrm{yr}$. If the radial velocity changes are due to orbital motion, the radius of the orbit is $\sim 0.3$ pc and the total mass of the system is $\sim 7 \times 10^{9} \mathrm{M}_{\odot}$. In the binary black-hole model the masses of the two holes are $\sim 2.2 \times 10^{9} \mathrm{M}_{\odot}$ and $\sim 4.4 \times 10^{9} \mathrm{M}_{\odot}$. A possible third peak claimed to be present in some 1974-75 spectra is shown to be an instrumental artifact. The narrowness of the displaced peaks in 3C 390.3 objects requires that the broad line region cloud motions are not governed solely by gravity. The black hole masses derived by Koratkar \& Gaskell (1991) need to be increased significantly. This probably lowers accretion efficiences to less than $1 \%$ of the Eddington limit.
\end{abstract}

Subject headings: accretion: accretion disks — black hole physics — galaxies: active - quasars: emission lines - quasars: general - quasars: individual (3C $390.3)$ 


\section{INTRODUCTION}

While most quasars (by which I mean all active galactic nuclei without regard to luminosity) have broad emission lines that are superficially symmetric, there is a class of quasars with broad lines showing one or two peaks Doppler shifted from the rest frame of the host galaxy by substantial amounts (Gaskell 1983). The first example discovered was 3C 390.3 (Sandage 1966; Lynds 1968) and, since it is also by far the best studied case, I will refer to quasars with displaced broad-line emission peaks as "3C 390.3 objects". I give their basic properties in Gaskell (1983, 1988b). For more details than can be discussed here, and for statistics of the occurence of 3C 390.3 quasars, I refer the reader to Gaskell (1996). The most spectacular cases tend to be radio-loud but there are also radio-quiet cases and I argue in Gaskell (1996) that displaced broad line peaks are very common, especially among quasars that are not seen close to face-on. The main models suggested to explain the displaced peaks include:

1. Bi-conical ejection. (Burbidge \& Burbidge 1971; Zheng, Binette \& Sulentic 1990; Zheng, Veilleux \& Grandi 1991) The main problem with ejection models (Oke 1987; Gaskell 1988b) is that 3C 390.3 objects tend not to be face-on. The gas motion is almost certainly perpendicular to the line of sight. Additionally, variability studies show that the motion of BLR gas is certainly not pure outflow (Gaskell 1988a; Koratkar \& Gaskell 1991a,b; Crenshaw \& Blackwell 1990; Maoz et al. 1991; Korista et al. 1995), although generalizing from these to 3C 390.3 objects might be unwise.

2. Supermassive Binary Black Holes. Gaskell (1983) suggested that 3C 390.3 quasars are "spectroscopic binaries" with each BLR peak being associated with its own black hole. The existence of binary black holes in quasars had been suggested by Begelman, Blandford \& Rees (1980) as an explanation of the apparent precession of radio jets. Since it is now clear that a large fraction of galaxies harbor massive black holes and that mergers between galaxies are common, many such binaries must form (a numerical simulation of the merger of the nuclei of two galaxies is well illustrated in the videotape of Barnes, 1992). The precession-like wiggling of radio-jets is well established (see Roos 1988 and Lu 1990 for extensive references). The binary black hole model gives a natural explanation for the magnitude of the velocity shifts of displaced peaks in 3C 390.3 quasars, for why blue and red peaks are equally common, for why some objects show one displaced peak while others show two, for why the broad lines are wider in 3C 390.3 objects, and for the variability of the peaks and continuum. These issues are all discussed at length in Gaskell (1996). 
3. Line emission from an accretion disk. Disk geometries for the BLR have been considered by many workers (Mathews 1982) A disk origin for the double peaks in 3C 390.3 objects was suggested by Oke (1987), Perez et al. (1988), and Halpern \& Filippenko (1988). There are many problems for disk models however (Mathews 1982; Mathews \& Capriotti 1985; Gaskell 1988b; Gaskell 1996). The most serious are that many double-peaked profiles cannot be fit by disk models and that the model cannot explain line profile variability (see Gaskell 1988b, 1996). In the disk model, both sides of the profile have to vary together in response to a change in the central ionizing continuum (Gaskell 1988b). They do not (Gaskell 1988b; Miller \& Peterson 1990). The predicted polarization is also not seen (Antonucci, Hurt \& Agol 1995).

The pros and cons of these and other models (e.g., the anisotropic continuum model of Wanders et al. 1995), and ways around these difficulties, are discussed in more detail in Gaskell (1996).

The supermassive binary model predicts that the wavelengths of the displaced peaks should change. Predicted orbital periods are of the order of centuries (see Gaskell 1983). Halpern \& Filippenko (1988) have argued that the apparent absence of a change in relative radial velocity in the $3 \mathrm{C} 390.3$ object Arp 102B rules out the binary black hole model, but this argument is based on the assumption of relatively low masses for the two black holes in Arp 102B. On the other hand, Veilleux \& Zheng (1991), have reported changes in the wavelength of the blue displaced peak in 3C 390.3. After a period when the blue peak almost completely vanished it (or another feature) reappeared at a longer wavelength. It is not clear, however, whether what was seen is a long period change or indeed whether it was a steady change in wavelength with time at all. For another object (OQ $208=\mathrm{Mrk}$ 668), Marziani et al. (1993) have claimed that the wavelength of a displaced peak changes with continuum luminosity rather than time. In this Letter I show that the wavelength of blue peak in 3C 390.3 does indeed change at a constant rate with time, as predicted by the binary black hole model in most cases, and that this change has no connection with line or continuum flux.

\section{DATA AND ANALYSIS}

The biggest published set of observations of 3C 390.3 are the Lick Observatory observations made by D. E. Osterbrock, J. S. Miller and their collaborators. These are presented in a very convenient format by Veilleux \& Zheng (1991). These spectra cover the 15 yr period 1974 - 1988. Up until mid-1984 the observations were made with various Robinson-Wampler Image Dissector Scanner (IDS) spectrograph systems; after mid-1984 
observations were made with a CCD spectrograph. The majority of the Lick spectra are of the $\mathrm{H} \beta$ region.

Veilleux \& Zheng (1991) only give eye estimates of the wavelength of the blue peak. I therefore measured all of the Lick spectra that showed a convincing blue displaced peak. A quasi-continuum was set under the displaced peak to allow for blending with the central (undisplaced) emission line. Rather than using the apparent "peak" of the displaced line, which is noise-sensitive, the Pogson method (Hoffmeister, Richter \& Wenzel 1985) was used to determine the position of each peak. Based on a comparison of spectra taken close together, the rms error for a single measurement was $\pm 2.0 \AA$ (versus $\pm 4.3 \AA$ for the Veilleux \& Zheng measurements). My measurements of the peak positions were systematically blueshifted by $4.0 \AA$ relative to the Veilleux \& Zheng eye estimates. During the "low state" of 3C 390.3 (circa 1980), when the blue peak was too weak to measure, I adopted the wavelengths of Veilleux \& Zheng, but blueshifted them by $4 \AA$ to put them on the same system as my measurements.

The 1965 photographic spectrum of Sandage (1966) has a small defect right where the center of the peak is predicted to be. It cannot be used for a precise measurement, but is useful for confirming that the blue peak existed 30 years ago. Lynds (1968) presents a number of photographic image-tube spectra taken in 1967. The positions of the blue peaks in these were measured in enlargements. The uncertainty of the average wavelength was calculated from the residuals of all lines from the wavelength calibration curve. Burbidge \& Burbidge (1971) give measurements of several 1970 Lick Observatory photographic image tube spectra. I adopted the mean $\mathrm{H} \beta$ blue peak wavelength from their table 1 . The error bar was calculated as for the Lynds (1968) spectrum.

3C 390.3 also shows a redshifted displaced peak which can be quite prominent at times (e.g., when the continuum and blue peak were faint in June/July 1980). It would obviously be interesting to also get relative radial velocities for the redshifted peak. There are two general problems however. First, this peak, unfortunately, is twice as close to the unshifted peak as the blue one is, so blending is more of a problem and the position depends on how the central peak is subtracted off. Second, there is also blending with the strong [O III] $\lambda 4959$ line. Nevertheless, Veilleux \& Zheng (1991) do tabulate eye estimates of wavelengths of the red peak. They indicate that these are highly uncertain. Because of the difficulty in measuring this peak, no attempt has been made here to measure the peak wavelengths and no radial velocity curve has been given.

Veilleux \& Zheng claim there is a third peak, redshifted by $+4600 \mathrm{~km} \mathrm{~s}^{-1}$, which is only visible in the Lick spectra of 1974-1975. If this were real it would pose a serious problem for many models. However, weak features in IDS spectra need to be treated with 
caution for a number of reasons. First, the IDSs were not photon-counting detectors. Each photon produced a different number of counts (see Robinson \& Gaskell 1978). Second, the illumination of the detector by the quartz lamp continuum source used to divide out small-scale response variations was frequently different than the the illumination by the target object. This could produce repeatable spurious features. Different observers used different slits, deckers and apertures, even on the same IDS. Finally, the IDS was heavily oversampled, so artificial bumps in spectra look smooth. Examination of the 3C 390.3 spectra shows that the "third" peak is almost certainly a result of the IDS instrumental problems. Rather than being a true emission feature, the $+4600 \mathrm{~km} \mathrm{~s}^{-1}$ feature is really the result of a sharp dip in the spectrum to the blue. This blue dip is most prominent in two spectra taken by the Miller group on June 4 and June 5 1975. The instrumental nature of this is demonstrated by it vanishing in a spectrum taken by the Osterbrock group with a different instrumental setup on the very next night.

\section{RESULTS}

The radial velocity curve for the blue peak is shown in Figure 1. Points are annual means. Error bars are $\sigma / \sqrt{ } n$. The open circles are median values (from Veilleux \& Zheng 1991) when the blue peak was essentially absent from the spectra. The latter have not been used in any further analysis.

The most striking result is the almost linear change in the radial velocity of the blue peak over more than two decades. The brightness of 3C 390.3 has varied a lot during this period and the character of the variability has changed as well. Shen, Usher \& Barrett (1972) report a change of 1.15 magnitudes in only 3 days, suggesting 3C 390.3 was in the

class of optically violent variables (OVVs) at that time. The high polarization also supports an OVV classification (see Angel \& Stockman 1980). The Herstmonceux monitoring of Lloyd (1984) shows a similarly rapid, though not quite as large, change in 1969 . The Herstmonceux light curve shows that 3C 390.3 was much brighter and more active around the time of the Lynds (1968) and Burbidge \& Burbidge (1971) spectra than during most of the Lick spectra. An important implication of Figure 1 is that the change in radial velocity is not a function of the continuum luminosity. Marziani et al. (1993) claimed that the wavelength of one of the displaced peaks in OQ 208 correlated with luminosity. This is clearly not the case with the blue peak in 3C 390.3 since the wavelength is increasing monotonically while the luminosity of both the continuum and the blue peak goes through a minimum around 1980. Since the offset peak in OQ 208 is blended with the central unshifted peak, I believe that the correlation Marziani et al. found is caused by changing 
blending with the central peak. The correlation they find is certainly in the right sense and of the right magnitude for this to be the cause. In 3C 390.3 it is obvious that the steady change in wavelength has persisted from when 3C 390.3 was bright and "active", through the "low state", around 1980 when the peak almost completely vanished, and on to the revival in the later 1980's.

We can draw at least two conclusions from this:

1. The gas producing the blue peak is long-lived. It is not some transient feature. The peak is wide enough that over $20 \mathrm{yr}$ it would have dissipated if the gas were not bound to something or if there were no source of gas.

2. The wavelength of the peak has nothing to do with the luminosity of the central engine. Since, as is normal for quasars, the total $\mathrm{H} \beta$ and $\mathrm{H} \alpha$ fluxes track the observed continuum luminosity (see Figure 1 of Oke 1987 and Figures 1a and 1b of Veilleux \& Zheng 1991), it is not possible to claim that the variability of the ionizing continuum emission is highly anisotropic and that the continuum variation observed on earth is radically different from that seen by the blue peak.

We can learn more from Figure 1 if we adopt the natural hypothesis that the change in radial velocity is the result of orbital motion of the gas producing the blue peak. The first step is to determine the orbital period. It is probably fairly safe to assume that the orbit is circular: dynamic friction will probably have accomplished this. Unfortunately, the period is not well constrained. A straight-line (infinite period) already has a $\chi^{2}$ per degree of freedom close to unity. It is possible, however, to set a lower limit on the period. A period of $210 \mathrm{yr}$ (the most extreme curve in Figure 1) is excluded at the $90 \%$ confidence level. A period of $300 \mathrm{yr}$ (the middle curve in Figure 1 ) is completely acceptable $\left(\chi^{2}\right.$ per d.o.f. $=$ 0.8). A period of $210 \mathrm{yr}$ gives a maximum observed velocity of $4700 \mathrm{~km} \mathrm{~s}^{-1}$; a period of 300 yr gives $5340 \mathrm{~km} \mathrm{~s}^{-1}$. For long orbital periods the maximum velocity scales approximately linearly with the orbital period. Since the observed relative velocity of the blue peak is already one of the largest known, orbital periods at the lower end of the acceptable range are favored. I will therefore adopt a period of $300 \mathrm{yr}$ for further calculation. The true period will probably be within $50 \%$ of this.

It is obvious from the curves in Figure 1 that it will be possible within a few years to constrain the orbital period quite tightly (possibly through the current International AGN Watch monitoring program). Measurement of possible additional archival 1960's spectra would also be helpful. The predicted date of zero relative radial velocity is insensitive to the period. If the period is $210 \mathrm{yr}$, zero relative radial velocity is predicted to occur in the year 2012; if the period is $300 \mathrm{yr}$, it will be in 2018 (the limiting case of an infinite period 
gives 2029). In the second decade of the 21 st century $3 \mathrm{C} 390.3$ should no longer be a $3 \mathrm{C}$ 390.3 object!

Adopting a $300 \mathrm{yr}$ period, the maximum velocity is $4700 \mathrm{~km} \mathrm{~s}^{-1}$. For a circular orbit this is $v_{\max } \sin i$, where $i$ is the inclination of the 3C 390.3 jets to our line of sight. This inclination can be estimated from the synchrotron self-Compton model and relativistic beaming considerations (Ghisellini et al. 1993). The ratio of optical flux to radio core flux supports such estimates (Wills \& Brotherton 1995). For 3C 390.3, Ghisellini et al. get $i \sim 29^{\circ}$. This gives $v_{\max } \sim 9400 \mathrm{~km} \mathrm{~s}^{-1}$. The radius of the orbit is therefore almost exactly one lt-yr $(0.3 \mathrm{pc})$ and the total mass is $6.6 \times 10^{9} \mathrm{M}_{\odot}$. The ratio of the relative radial velocity shifts of the blue and red peaks is 2:1 if we assume that the narrow lines

indicate the combined center of mass, so the masses of the black holes are $4.4 \times 10^{9} \mathrm{M}_{\odot}$ and $2.2 \times 10^{9} \mathrm{M}_{\odot}$.

If the binary black hole model is correct, the redshifted peak should be showing the opposite motion to the blueshifted peak. As mentioned in the previous section there are major difficulties in determining the relative radial velocity of the red peak, but the changes in the wavelength of the red peak do indeed seem to be consistent with the predictions of the binary model. Over the last 10 years of the Lick data, the magnitude of the relative radial velocity of the red peak has decreased by many hundreds of $\mathrm{km} \mathrm{s}^{-1}$.

\section{DISCUSSION}

Although the black hole masses found are in agreement with the masses claimed for black holes in nearby galaxies (e.g., the $H S T$ determination of $2.4 \times 10^{9} \mathrm{M}_{\odot}$ in $\mathrm{M} 87$; Ford et al., 1994), the black holes masses found are somewhat higher than predicted by the Koratkar \& Gaskell (1991b) mass-luminosity relationship. A possible reason for this is easy to understand. Koratkar \& Gaskell obtained their quasar mass estimates from velocity dispersions and size estimates from their "reverberation mapping". Gaskell (1988a) had also shown that the motion of BLR gas is not predominantly radial, and Koratkar \& Gaskell (1991a) found this to be generally true of the quasars they considered. They therefore assumed that the motions were gravitationally dominated and used the virial theorem to find the masses. Penston (1987) pointed out that gravitationally dominated BLR motion was incompatible with the binary black hole model because one needs velocity dispersions lower than the orbital velocity for gas bound to each black hole (for discussion of this point see footnote 3 of Cheng, Halpern \& Filippenko 1989). There is a natural solution to this apparent dilemma: unlike black holes, BLR clouds can easily be influenced by non-gravitational forces. Both gravity and radiation pressure are inverse square law forces. 
Clouds can orbit under the combined force. The mean velocity at a given radius from a black hole will be less than the orbital velocity of particles subject only to gravity. If one assumes that the BLR clouds are subject only to gravity a serious underestimate of the black hole mass results. There is every reason to believe that radiation pressure and wind pressures are very significant and that in some situations they can even exceed gravity, since at least some gas is being seen to be expelled at high velocities from quasars (the broad absorption line gas). A more complicated model invoking both radiation pressure and gravity has been presented by Mathews (1993). He estimates that Koratkar \& Gaskell (1991b) have underestimated black hole masses by a factor of about 10-20.

If this reconciliation of reverberation mapping results with the binary black hole model is correct, then the Eddington efficiences found by Koratkar \& Gaskell (1991b) have also been overestimated. Quasar black hole accretion efficiences fall from a few percent to a few tenths of a percent.

The size of the orbit is only a few times the size of the BLR inferred from reverberation mapping. Obviously cloud motions in the presence of two black holes are complicated and detailed modelling work is needed.

The spin axis of a rotating orbiting black hole can be subject to geodetic precession. Given the masses and orbital sizes, the precession period, $P_{p r e c}$, can be derived (see equation 8 of Begelman, Blandford \& Rees 1980). For the parameters just inferred from the 3C 390.3 radial velocity curve, $P_{\text {prec }} \sim 4 \times 10^{5} \mathrm{yr}$. This is very typical of precession periods deduced from wiggles in quasar jets (see Roos 1988 and $\mathrm{Lu}$ 1990). The jet of 3C 390.3 is straight however, at least on kpc scales. This implies that, in this case, the spin and orbital angular momentum vectors are closely parallel.

Although the simple disk model of 3C 390.3 objects has been strongly ruled out for a long time (see introduction), there have been modifications to the model. Zheng, Veilleux \& Grandi (1991) introduced "hot spots" on the disk (there is precedence for this from cataclysmic variable star work). The radial velocity change in Figure 1 is compatible with a hot spot orbiting one black hole, but such a model does not explain why the blue peak is correlated with the continuum changes while the red one is not, or indeed why the profile should change at all. Eracleous et al. (1995) introduced elliptical accretion disks to explain some profiles not fit by simple accretion disk models. In these models the observed radial velocity change would be due to relativistic precession and tidal effects of a binary companion black hole. The period is about right, but, as Eracleous et al. concede, this model does not explain changes in the intensity ratios of the red and blue peaks. Chakrabarti \& Wiita (1994) propose that the humps are due to spiral shocks in accretion discs. Unfortunately, they predict a period of $21 \mathrm{yr}$ for 3C 390.3, which is too short by 
at least a factor of 10, and, once again, the model fails to explain the line profile and continuum variability.

\section{CONCLUSIONS}

The observations analyzed here present strong support for the binary black hole model. One of the major predictions of the model, a change in radial velocity over time, has been accurately confirmed. The initial failure to detect such a change in Arp 102B is not a problem if Arp 102B has black hole masses as large as in 3C 390.3 or in a typical remnant. It is possible that the radial velocity changes seen in Figure 1 are produced by a "hot spot"

or irregularity in a disk structure, but a number of factors (the shape of the line profile and the nature of the line variability in 3C 390.3 itself, and 3C 390.3 objects in general) argue strongly against this model. The production of supermassive black hole binaries through galactic mergers is something we know must be going on. 3C 390.3 objects seem to be a likely consequence. Clearly it will be interesting to follow other 3C 390.3 objects to see whether they too show radial velocity variations similar to those found in 3C 390.3 itself. Sergeev et al. (1994) have already claimed a velocity change of $2400 \mathrm{~km} \mathrm{~s}^{-1}$ in $\sim 800 \mathrm{~d}$ in NGC 5548.

I am grateful to Ski Antonucci, Mike Eracleous, Don Osterbrock, Sylvain Veilleux, Ignaz Wanders, and Wei Zheng for discussion of this work. 


\section{REFERENCES}

Angel, J. R. P. \& Stockman, H. S. 1980, ARA\&A, 8, 321

Antonucci, R. R. J., Hurt, T. \& Agol, E. 1995, ApJ Letters, in press

Barnes, J. E. 1992, ApJ, 393, videotape segment 2

Begelman, M. C., Blandford, R. D., \& Rees, M. J. 1980, Nature, 287, 307

Burbidge, E. M. \& Burbidge, G. R. 1972, ApJ, 163, L21

Chakrabarti, S. K. \& Wiita, P. J. 1994, ApJ, 434, 518

Cheng, K., Halpern, J. P. \& Filippenko, A. V. 1989 ApJ, 339, 742

Crenshaw, D. M. \& Blackwell, J. H., Jr. 1990, ApJ, 358, L37

Eracleus, M., Livio, M., Halpern, J. P., \& Storchi-Bergmann, T. 1995, ApJ, 438, 610

Ford, H. C. et al. 1994, ApJ, 435, L27

Gaskell, C. M. 1983 in Quasars and Gravitational Lenses, 24th Liege Astrophysical Colloquium, 471

Gaskell, C. M. 1988a, ApJ, 325, 114

Gaskell, C. M. 1988b, in Active Galactic Nuclei, ed. H. R. Miller \& P. J. Wiita (Berlin: Springer), 61

Gaskell, C. M. 1996, in Jets from Stars and Active Galactic Nuclei, ed. W. Kundt, (Berlin: Springer), 165 (astro-ph/9605175)

Ghisellini, G., Padovani, P., Celotti, A., \& Maraschi, L. 1993, ApJ, 407, 65

Halpern, J. P. \& Filippenko, A. V. 1988, Nature, 331, 46

Hoffmeister, C., Richter, G., \& Wenzel, W. 1985, Variable Stars (Berlin: Springer), 280

Koratkar, A. P. \& Gaskell, C. M. 1991a, ApJS, 75, 719

Koratkar, A. P. \& Gaskell, C. M. 1991b, ApJ, 370, L61

Korista, K. T., et al. 1995, ApJS, 97, 285

Lloyd, C. 1984, MNRAS, 209, 697

Lu, J. J. 1990, A\&A, 229, 424

Lynds, C. R. 1968, AJ, 73, 888

Marziani, P., Sulentic, J. W., Calvani, M., Perez, E., Moles, M. \& Penston, M. V. 1993, ApJ, 410, 56 
Maoz, D., Netzer, H., Mazeh, T., Beck, S., Almoznino, E., Leibowitz, E., Brosch, N., Mendelson, H. \& Laor, A. 1991, ApJ, 367, 493

Marziani, P., Sulentic, J. W., Calvani, M., Perez, E., \& Penston, M. V. 1993, ApJ, 410, 56

Mathews, W. G. 1982, ApJ, 258, 425

Mathews, W. G. 1993, ApJ, 412, L17

Mathews, W. G. \& Capriotti, E. R. 1995, in Astrophysics of Active Galaxies and Quasi-Stellar Objects, ed. J. S. Miller (Mill Valley: University Science Books), 185

Miller, J. S. \& Peterson, B. M. 1990, ApJ, 361, 98

Oke, J. B. 1987, in Superluminal Radio Sources, ed. J. A. Zensus \& T. J. Pearson (Cambridge: Cambridge Univ. Press), 267

Penston, M. V. 1987, private communication

Perez, E., Penston, M.V., Tadhunter, C., Mediavilla, E., \& Moles, M. 1988, MNRAS, 230, 353

Robinson, L. B. \& Gaskell, C. M. 1978, in 7th Symposium on Photoelectronic Imaging Devices (London: Imperial College), 103

Roos, N. 1988, ApJ, 334, 95

Sandage, A. R. 1966, ApJ, 334. 95

Sergeev, S. G., Malkov, Yu. F., Chuvaev, K. K., \& Pronik, V. I. 1994, in Reverberation Mapping of the Broad Line Region in Active Galactic Nuclei, ed. P. M. Gondhalekar, K. Horne, \& B. M. Peterson (San Francisco: Astronomical Society of the Pacific), 199

Shen, B. S. P., Usher, P. D. \& Barrett, J. W. 1972, ApJ, 171, 457

Veilleux, S., \& Zheng, W. 1991, ApJ, 377, 89

Wanders, I., et al. 1995, ApJ 453, L87

Wills, B. J. \& Brotherton, M. S. 1995, ApJ, 448, L81

Zheng, W., Binette, L., \& Sulentic, J.W. 1991, ApJ, 365, 115

Zheng, W., Veilleux, S., \& Grandi, S.A. 1991, ApJ, 381, 418 


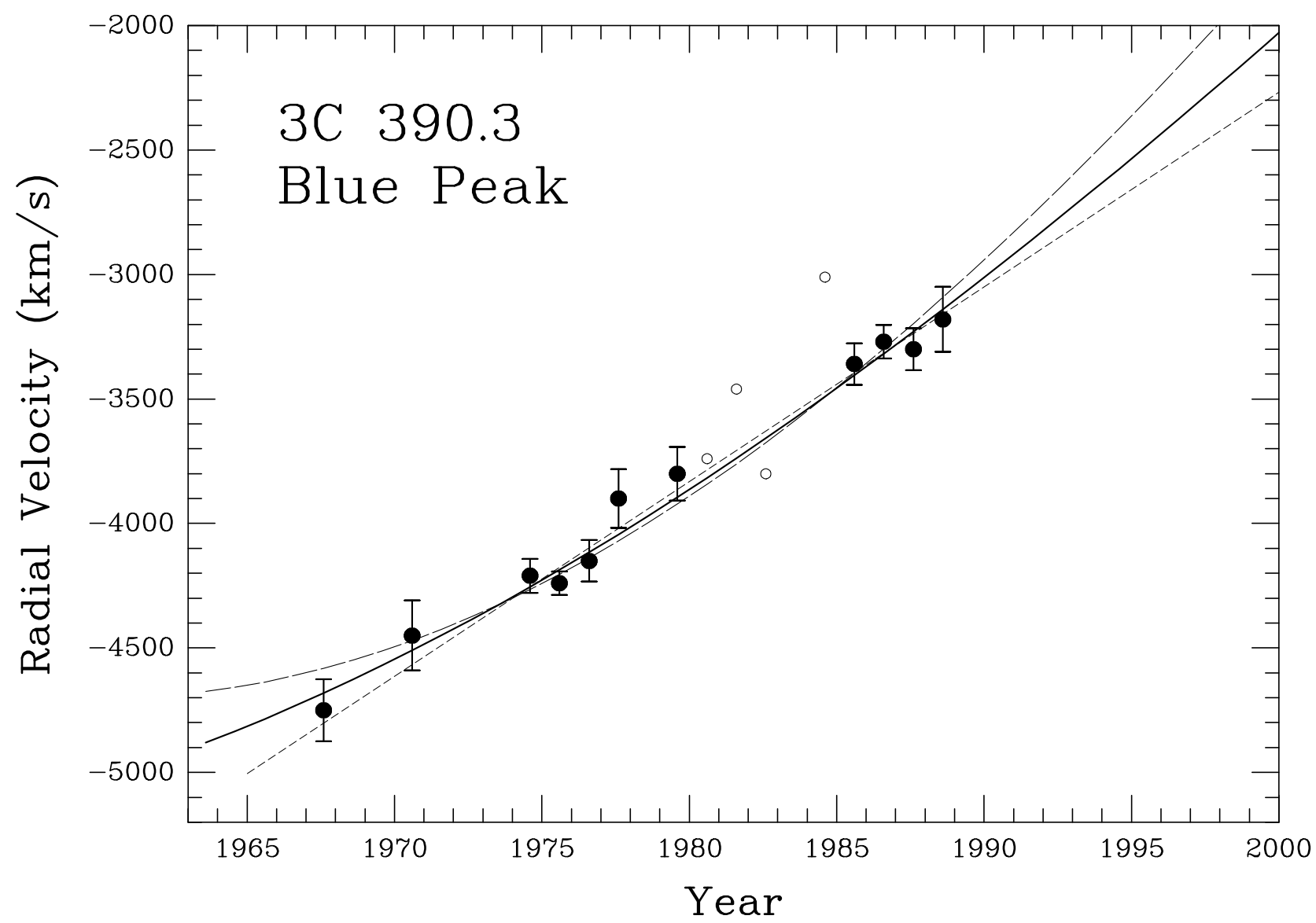

Fig. 1. - Radial-velocity curve for the blue displaced broad peak of $\mathrm{H} \beta$ in $3 \mathrm{C}$ 390.3. Error bars are calculated as described in the text. The open circles are median values from the eye estimates of Veilleux \& Zheng (1991) when the blue peak was essentially absent from the spectra. The long-dashed curve is for a $210 \mathrm{yr}$ period (excluded at the $90 \%$ confidence level), the solid curve is for a $300 \mathrm{yr}$ period and the straight line is the limiting case of an infinite period. 